\title{
Recurrent spontaneous multiple pregnancy: a case report
}

\author{
Shazia Parveen*, Noor Afshan Sabzposh, Amina Kuraishy
}

Department of Obstetrics and Gynaecology, J. N. Medical College, Aligarh Muslim University, Aligarh, UP, India

Received: 03 June 2016

Accepted: 01 July 2016

*Correspondence:

Dr. Shazia Parveen,

E-mail: shzparveen@gmail.com

Copyright: () the author(s), publisher and licensee Medip Academy. This is an open-access article distributed under the terms of the Creative Commons Attribution Non-Commercial License, which permits unrestricted non-commercial use, distribution, and reproduction in any medium, provided the original work is properly cited.

\begin{abstract}
Multiple gestations are usually iatrogenic like use of assisted reproductive techniques (ART), infertility treatment but it is rare in spontaneous conception. High order multiple pregnancies (HOMPs) are major cause of maternal, fetal and neonatal morbidity. Multiple gestations carries 2 complications either abortion in early gestation or a preterm delivery in late pregnancy (more common). Preterm delivery is common (50\%) and patient usually delivers by 30-32 weeks. Discordance of fetal growth is very common and even more than in twins. Perinatal loss is inversely related to birth weight. The mothers should be counseled about regular ante natal care (ANC) check-ups for early identification of multiple pregnancies so that proper care can be given to prolong the gestational age and reduce the complications associated with multiple pregnancies.
\end{abstract}

Keywords: Multiple pregnancies, ART, Spontaneous conception, ANC

\section{INTRODUCTION}

Multiple gestations are usually iatrogenic like use of ART, infertility treatment but it is rare in spontaneous conception. Its incidence is becoming a problem of increasing dimension because of wide spread use of assisted reproductive techniques. ${ }^{1}$ The incidence decreases as the number of fetuses increases. According to Hellins law, incidence of twin is 1:80, triplet is $1: 6400$ whereas that for quadruplets is approximately 0.000142 i.e. less than $1 / 700,000$ singleton births, twins, triplets and quadruplets may develop from fertilization of single or 2 or even 3 ovums. Multiple pregnancies are associated with many complications like miscarriages, preterm labor, pre eclampsia, placenta previa etc. ${ }^{3}$ The gestational age at delivery decreases with the number of fetuses. The mean gestation age of twins is 35.3 weeks where as that of quadruplets is 29.3 weeks. More than 98\% quadruplets will be born before 37 weeks and will be low birth weight i.e., less than $2500 \mathrm{gm}$. The weight of the new born also decreases with the increase in number of fetuses. Here we report a rare case of spontaneous quadruplet pregnancy where mother had delivered a twin two years ago. In both pregnancies the conceptions were spontaneous without any fertility treatment.

\section{CASE REPORT}

Mrs. A, 25 years, $\mathrm{G}_{2} \mathrm{P}_{1+0} \mathrm{~L}_{0}$ rural based female married since 5 years, presented as emergency case with 26 weeks pregnancy with bleeding per vagina (BPV) for 3 hours with shock. She had one preterm vaginal home delivery of twins at 7 month of gestation age, 2 years ago. Both died after 24 hours of birth. There was no history of any infertility treatment or any ovulation induction drugs taken for conception in any of the two pregnancies.

On examination she was severely pale (clinically 3-4 $\mathrm{gm} \%), \mathrm{PR}=108 \mathrm{~min}, \mathrm{BP}=80 \mathrm{mmHg}$ systolic. On P/Auterus 34 weeks, exact presentation not made out, FHS not localized. On local examination - BPV present. Her $\mathrm{Hb}$ was $2.2 \mathrm{gm} \%$, platelet count $=48,000 / \mathrm{mm}^{3}$. On P/V examination, os was $6 \mathrm{~cm}$, no placenta felt. Spontaneous rupture of membrane occurred and 4 dead babies delivered vaginally. First delivered by vertex and rest 
three delivered by breech. Two of them were female weighing $500 \mathrm{gm}$ and $410 \mathrm{gm}$. Other two were male weighing $450 \mathrm{gm}$ and $400 \mathrm{gm}$ respectively. There were 4 placentas and 4 umbilical cords. Although it is rare, most probable reason could be fertilization of 2 ova by 2 sperms. Further division of 2 zygotes into 4 zygotes and 4 small gestational sacs. All due precautions to prevent PPH were taken. Patient was transfused two units of blood. She was discharged after seven days in good condition.

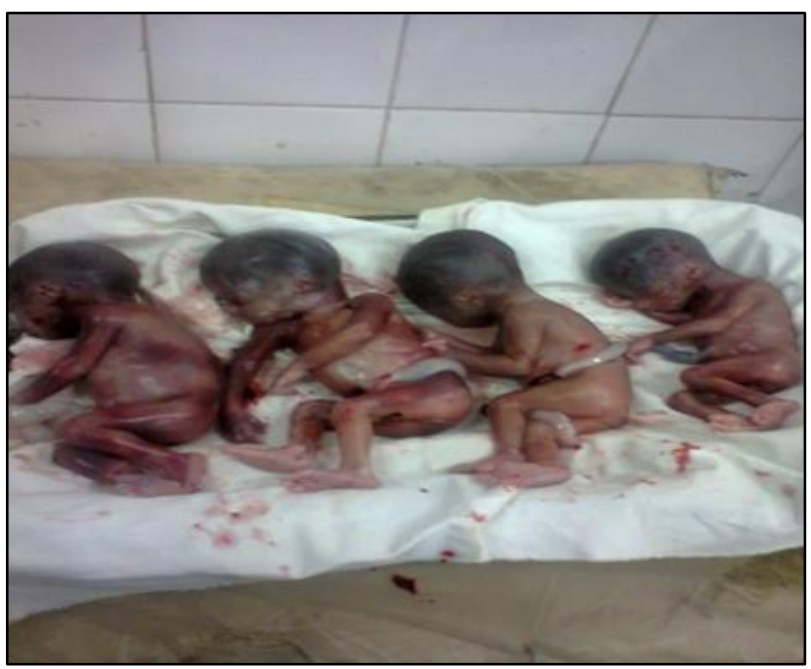

Figure 1: Quadruplets with four separate placentas.

This was rarest case of delivering multiple recurrent babies with four separate placentas, after purely natural and spontaneous conception without any iatrogenic intervention.

\section{DISCUSSION}

A retrospective audit was performed of all high order multiple pregnancies (HOMP's) delivered in various maternity hospitals in different countries for a 10 year period. A total of 101 HOMP's occurred, 95 triplets, 7 quadruplets and 1 quintuplet. Information regarding mode of conception was available for $81 \%$ pregnancies.
Only about one third viz., 28\% were conceived spontaneously, rests were after ART. ${ }^{4}$ So from all available data we conclude that majority multiple pregnancies occur due to iatrogenic, arising from fertility treatment including clomiphene/gonadotropins, natural mode of conception is very rare. In general, diagnosis is usually accidental following sonography during birth. Triplets and HOMP's are major cause of maternal, fetal and neonatal morbidity. Multiple gestations carries two complications either abortion in early gestation or a preterm delivery in late pregnancy (more common). Preterm delivery is common $(50 \%)$ and patient usually delivers by 30-32 weeks. Discordance of fetal growth is very common and even more than in twins. Perinatal loss is inversely related to birth weight.

\section{CONCLUSION}

The mothers should be counselled about regular ANC check-ups for early identification of multiple pregnancies so that proper care can be given to prolong the gestational age and reduce the complications associated with multiple pregnancies.

\section{Funding: No funding sources Conflict of interest: None declared Ethical approval: Not required}

\section{REFERENCES}

1. Spectn TD, Williams FM. UK audit twin register. Twin Res Hum Genet. 2006;9(6):899-906.

2. Lam H, Ho PC. A comparison of pregnancy outcome between high order multiple and twin pregnancy; matched pair retrospective study. HKMJ. 1999;5:1620.

3. Norwitz ER, Edusa V, Park JS. Maternal physiology and complication of multiple pregnancy. 2005;29(5):338-48.

4. Basit I, Johnson SN, Geary M, Daly S, Wingfield M. Mode of conception of triplets and high order multiple pregnancies. Ireland Med Journal. 2012;105(3):80-3.

Cite this article as: Parveen S, Sabzposh NA, Kuraishy A. Recurrent spontaneous multiple pregnancy: a case report. Int J Reprod Contracept Obstet Gynecol 2016;5:3208-9. 